\title{
The heart of the swarm: K2 photometry and rotational characteristics of 56 Jovian Trojan asteroids
}

\author{
Gy. M. Szabón ${ }^{1,2}$, A. Pál ${ }^{2,3}$, Cs. Kiss², L. L. Kiss ${ }^{2,4}$, L. Molnár², O. Hanyecz²,3, \\ E. Plachy ${ }^{2}$, K. Sárneczky², and R. Szabó \\ 1 ELTE Gothard Astrophysical Observatory, 9704 Szombathely, Szent Imre herceg útja 112, Hungary \\ e-mail: szgy@gothard.hu \\ 2 Konkoly Observatory, MTA Research Centre for Astronomy and Earth Sciences, Konkoly-Thege Miklós út 15-17, 1121 Budapest, \\ Hungary \\ e-mail: apal@szofi.net \\ 3 Department of Astronomy, Loránd Eötvös University, Pázmány Péter sétány 1/A, 1117 Budapest, Hungary \\ ${ }^{4}$ Sydney Institute for Astronomy, School of Physics A28, University of Sydney, NSW 2006, Australia
}

Received 26 July 2016 / Accepted 14 September 2016

\begin{abstract}
We present fully covered phased light curves for 56 Jovian Trojan asteroids as observed by the K2 mission of the Kepler space telescope. This set of objects has been monitored during Campaign 6 and represents a nearly unbiased subsample of the population of small solar system bodies. We derived precise periods and amplitudes for all Trojans, and found their distributions to be compatible with the previous statistics. We point out, however, that ground-based rotation periods are often unreliable above $20 \mathrm{~h}$, and we find an overabundance of rotation periods above $60 \mathrm{~h}$ compared with other minor planet populations. From amplitude analysis we derive a rate of binarity of $20 \pm 5 \%$. Our spin rate distribution confirms the previously obtained spin barrier of $\sim 5 \mathrm{~h}$ and the corresponding $\sim 0.5 \mathrm{~g} \mathrm{~cm}^{-3}$ cometary-like density limit, also suggesting a high internal porosity for Jovian Trojans. One of our targets, asteroid 65227 exhibits a double rotation period, which can either be due to binarity or the outcome of a recent collision.
\end{abstract}

Key words. minor planets, asteroids: general

\section{Introduction}

Trojan asteroids are located at a heliocentric distance of $\sim 5.2 \mathrm{AU}$ in the L4 and L5 Lagrange points of the Sun-Jupiter system (1:1 mean-motion resonance). Trojans were traditionally thought to be formed near to their present location, but recently, different scenarios were proposed for their origin. As indicated by the surface composition, they may have formed in the proto-Kuiper belt, then scattered inward, and been captured (the Nice model, e.g., Morbidelli et al. 2005; Tsiganis et al. 2005; Gomes et al. 2005; Levison et al. 2011) as a result of resonant interactions with the giant planets (e.g., Grav et al. 2011; Emery 2016). The "jump" scenario assumes the collisional scattering with Jupiter only, due to close interactions with an ice giant (Nesvorný et al. 2013). Due to their stable resonance locking, collisional frequencies are the lowest within these groups in the inner solar system (Dahlgren 1998; Dell'Oro 2001).

While dynamical arguments support the capture of Trojans from the outer solar system via collisional scattering, there are significant differences between the Trojans and the Centaurs or trans-Neptunian objects, indicating a possible surface modification due to inward migration. The photometric and spectroscopic data revealed the presence of two main taxonomical groups in the Trojan swarms, recognized as $\mathrm{D}$ and $\mathrm{P}$ in the Tholen (1984) classification. Both types exhibit featureless spectra, while D type has a redder slope and includes the majority of the Trojans. Unlike the Main Belt, the Trojan taxonomical types are not separated in the orbital elements space, but there is an inclination-color gradient present at least in the L4 swarm (e.g., Szabó et al. 2007), which represents taxonomical subgroups (Roig et al. 2008).

The bimodality of Jovian Trojan asteroids was also identified in their magnitude distribution (especially at the faint-end), in near-infrared spectra, infrared albedo, and also in colors, suggesting the existence of two distinct groups among Jovian Trojans (Wong et al. 2014, and references therein). Since this bimodality extends to several parameters, the presence of "red" and "less red" populations are suggested, with different origin and different evolution, instead of simply different taxonomical types which were mixed together (Wong et al. 2014; Wong \& Brown 2015). However, objects in these populations are still significantly bluer than the typical "red" objects in the Centaur and trans-Neptunian populations (Peixinho et al. 2012; Lacerda et al. 2014). A common, outer solar system origin of Jovian Trojans and trans-Neptunian objects was recently proposed by Wong \& Brown (2016), also suggesting that the retention or loss of $\mathrm{H}_{2} \mathrm{~S}$ in the early solar system was the likely reason behind the color differences we observe today.

In addition to the photometric characteristics discussed above, light curves available for Jovian Trojans can provide shape and rotational frequency distributions, and also information on the binary fraction. These statistics can be compared with the prediction of various formation and evolution models. In this sense, binary or multiple systems especially important, as their observations provide reliable masses and densities, a key to composition and internal structure. There are several formation mechanisms proposed for multiple systems (see, e.g., Merline et al. 2002; Noll et al. 2008), therefore their properties give an 
important clue on accretional, collisional and radiative processes as well, and may lead to the identification of differences between the red and the less red groups in the case of Jovian Trojans. A Wide-field Infrared Survey Explorer (WISE) survey found that $20 \%$ of Jovian Trojan asteroids are either extremely elongated objects, or are binaries (Sonnett et al. 2015).

In the $\mathrm{K} 2$ mission of the Kepler space telescope (Howell et al. 2014), 56 Jovian Trojan asteroids have been observed in Campaign 6, and long, uninterrupted light curves have been taken that are free from aliases, giving a more comprehensive description of these populations then the sparsely sampled WISE or ground based data. In this paper, we present the light curves and photometric properties of these 56 Jovian Trojan asteroids, all orbiting at the L4 Lagrange point of the Sun-Jupiter system. In Sect. 2 we summarize the observations and the data reduction schemes used to obtain the light curves of these Trojan asteroids. In Sect. 3 we present the statistical properties of this sample. Our results are summarized in Sect. 4. Tabulated data and the light curves of individual Trojans are shown in Appendix A similar study about Main Belt asteroids with K2 will be published in a related paper (Szabó et al. 2016).

\section{Observations and data reduction}

\subsection{K2 observations}

Kepler observed parts of the apparent trajectories of 56 Trojans during K2 Campaign 6, between 2015 July 13.95 and 2015 September 30.87 (UTC). In total, Campaign 6 data are formed from 3686 long cadence frames and the stamps allocated for these 56 Trojan asteroids have also been retrieved in long cadence mode, corresponding to a 29.41-min sampling frequency. To date, all of the moving objects observed by K2 were transNeptunian objects (Pál et al. 2015, 2016; Kiss et al. 2016) whose apparent speeds in the K2 CCD frames were so small that during the photometric analysis, these objects can be treated as point sources on the individual long cadence frames. However, Trojans of Jupiter are located significantly closer to the inner solar system, that is, to $\mathrm{K} 2$ itself. Hence, the apparent speed can exceed $0.1^{\prime \prime} / \mathrm{min}$, yielding trail-like corresponding PSFs on the long cadence frames. As we will describe later on (Sect. 2.2), the photometry of these trails requires non-circular apertures. The main parameters of the 56 observed Trojans are summarized in Table A.1. The apparent trajectories of these objects with respect to the K2 CCDs are shown in Fig. 1.

\subsection{Data reduction and photometry}

In principle, the reduction of K2 long cadence data corresponding to Jupiter Trojans follows similar steps to those used in earlier analyses of moving objects observed with K2 (Pál et al. 2015; Kiss et al. 2016). In order to correct for the positioning jitter of the spacecraft, we retrieved nearly a dozen additional stellar sources for each Trojan and included these in further processing (see e.g. Fig. 1 of Pál et al. 2016). While all of these 56 objects have been observed in the same campaign of $\mathrm{K} 2$, the determination of the positioning jitter has been done separately for each object. This is essential due to the large field-of-view: namely, the same pitch, roll and yaw offsets applied during the attitude control of the space telescope yields different apparent centroid shift and field rotation on the various CCD modules.

After the derivation of the positioning jitter of the subsequent frames, we registered the frames to the same reference system in order to perform differential image analysis. A series of frames
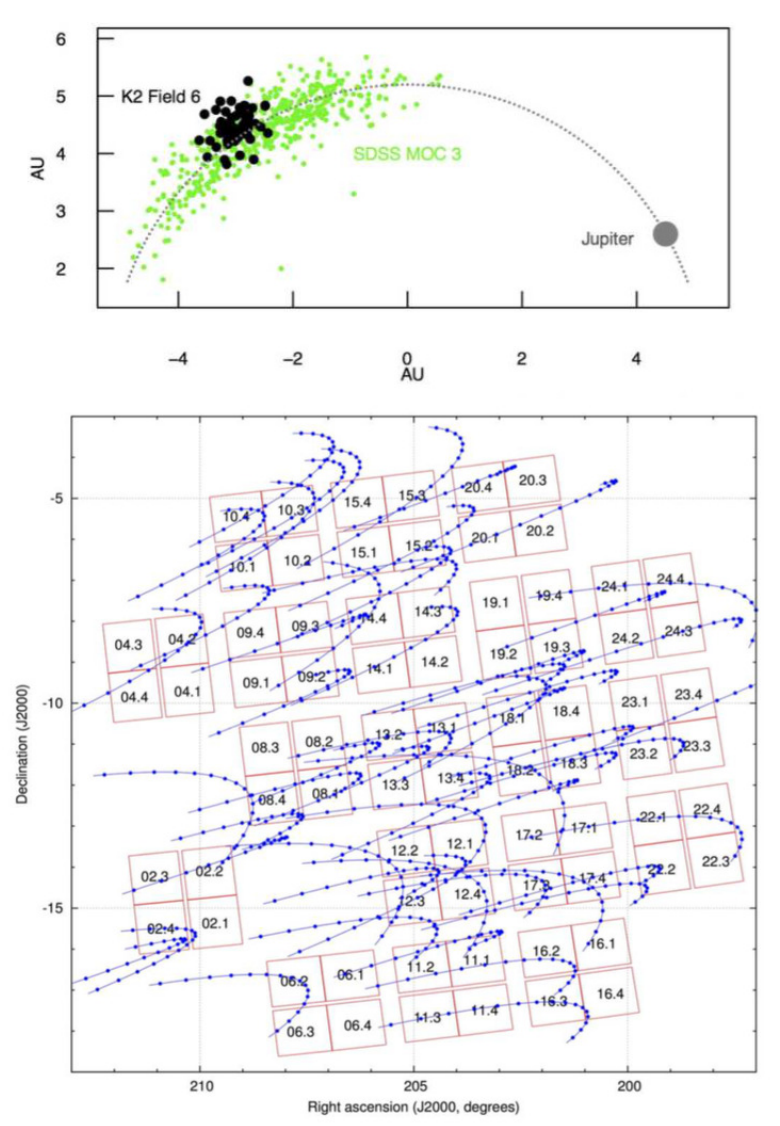

Fig. 1. Upper panel: upper view of Field 6 Trojans (large black dots) superimposed to the SDSS MOC3 Trojans in the L4 swarm (smaller grey dots) projected to the Jupiter's orbital plane. Earth is in origin. Note that Campaign 6 pointed exactly into the core of the L4 swarm. Lower panel: field-of-view of K2 Campaign 6 superimposed with the apparent trajectories of the 56 Jupiter Trojans discussed in this paper. On each trajectory, the dots show the motion in 5-days long steps.

were pre-selected to form a master median-combined image which was then subtracted from the subsequent frames. Differential aperture photometry was then performed using apertures that follow the expected shape of the apparent elongated PSFs of these objects. The apparent speed of $\lesssim 0.1^{\prime \prime} / \mathrm{min}$ mentioned above is equivalent to $\lesssim 2$ Kepler CCD pixels per long cadence sampling. Therefore, it is essential to take into account these shapes during the photometry. In Fig. 2 we display a short series of subsequent image stamps and the related apertures corresponding to (12974) Halitherses. This object showed the largest apparent speed, namely 1.9 ..2.1 pixels per long cadence frame. We note that this algorithm of using elongated apertures for flux extraction yields the same output in the limit of circular apertures at low apparent speed.

During the differential image procedures, the background stars cannot be completely eliminated. This is mostly due to the residual structures yielded both by the interpolation algorithm as well as the intrinsic photon noise of the stars themselves. These residual structures caused outlier points which were expunged from the final light curves. Light curve point exclusion was also caused by data loss during the telemetry of $\mathrm{K} 2$ data. On average, $\sim 10 \%$ of the data points were excluded from the light curves due to the aforementioned reasons. In Fig. 3 we plotted some corresponding statistical properties while quantities related to the light curve statistics are also found in Table A.1. 

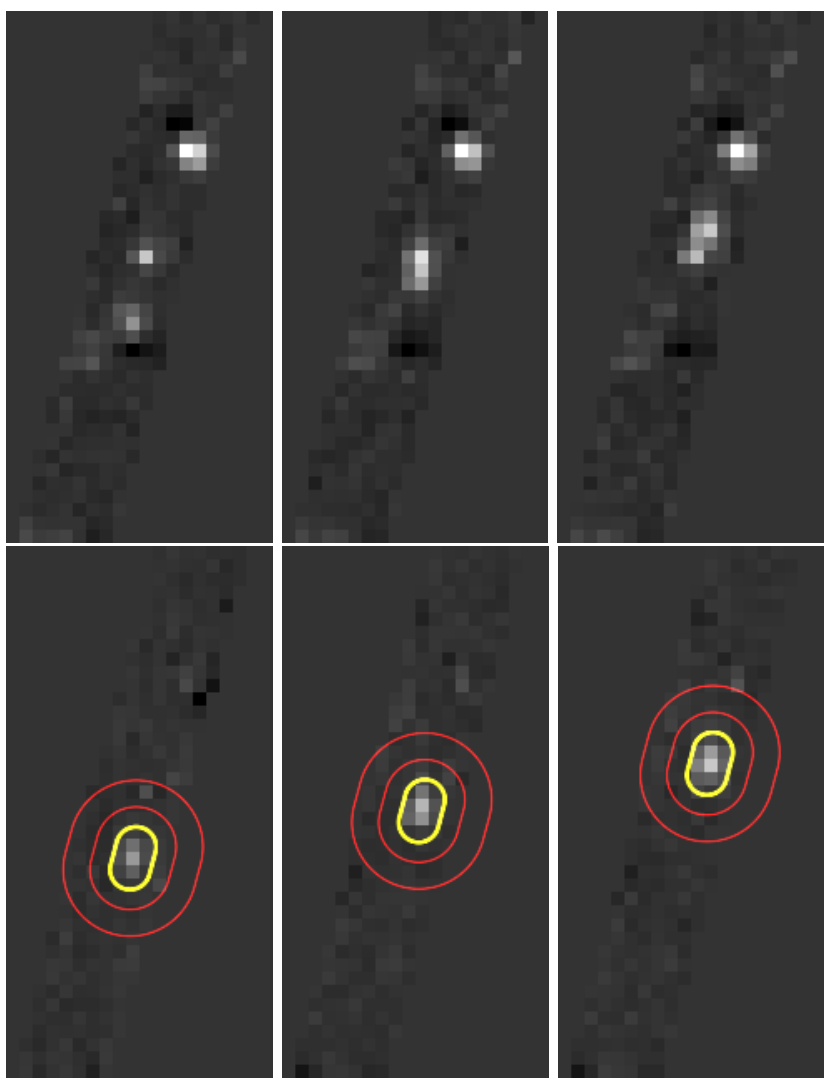

Fig. 2. Typical stamps taken from the image series related to (12974) Halitherses, which was the object with the fastest apparent speed (exceeding 2 pixels per long-cadence frame). The upper row of image stamps shows the original frames between the cadences \#2633 and \#2637 while the lower row shows the registered and differential stamps corresponding to their respective upper counterparts. The elongated aperture (bounded by the thick yellow curve) and the elongated annuli (bounded by the red curves) are used for the photometry and for the estimation of the background level, respectively. These stamps show a region of $20 \times 40$ pixels, i.e. an area of $1.33^{\prime} \times 2.66^{\prime}$ on the sky.

For all of the previously discussed photometric procedures, we applied the various tasks of the FITSH package (Pál 2012). We note here that the features of aperture photometry on noncircular (in fact, arbitrarily-shaped) apertures are included only in the upcoming, 0.9.2 version of the package. This new version is currently under preparation for public release and expected to be published on the website of the software ${ }^{1}$ soon.

The statistics of light curve acquisition are plotted in Fig. 3. The left panel shows the distribution of the apparent trail lengths of the Trojans on the long-cadence frames. It can easily be seen that one-third of the objects have an average trail length less than 0.5 pixels (which yields roughly indistinguishable PSF from the fixed sources), while two-thirds of the objects have a trail length less than one pixel. In addition, there are only two objects for which the trail length is larger than two pixels on average. The middle panel shows the distribution of the light curve coverages (i.e., the time span between the first and last light curve data point). Most of our objects were observed for 10-20 days, with a distinct peak around ten days, and there is another group scattering around 20 days of coverage. The right panel shows the number of light curve points as the function the duty cycle (percentage of the number of observed 29.4-min cadences with

\footnotetext{
http://fitsh.szofi.net/
}

respect to the maximum possible during the length of observation), that is, how many cadences have been lost due to technical problems originating mainly from the photometric pipeline (outliers, encountering too bright stars, stellar residuals, etc.) The thick blue line corresponds to the slope of $\approx 1 / 0.02043$ frame/day (where $0.02043 \mathrm{~d}$ is the $\mathrm{K} 2$ long cadence period) and the thin dashed blue lines show 90\%, 80\%, 70\% and 60\% duty cycles. The vast majority of our targets were observed with a duty cycle between 80 and $96 \%$, a handful of them above $70 \%$. The long coverage and the relatively continuous observations support the conclusion that period biases and aliases due to interruptions can only marginally affect our sample, if they bias it at all.

Of course our sample is magnitude limited, but is quite close to being complete in the observed magnitude range. In Szabó et al. (2015) we demonstrate that no undiscovered asteroids are found in the full-frame and part-frame $\mathrm{K} 2$ observations, confirming that the asteroids have been discovered down to the sensitivity limit of K2. Therefore we do not expect severe biases due to undetected small asteroids.

\subsection{Period analysis}

We searched for significant periodicities using the Fourier method as implemented in the Period04 program package (Lenz \& Breger 2005), and also the Lomb-Scargle periodogram in the gatspy Python package ${ }^{2}$. We got very similar results in several test cases, therefore we decided to stick to the LombScargle periods. We note that the errors of the individual photometric points are taken into account. Only those signals were considered that were significant on the $3 \sigma$-level compared to the background local noise periodogram. We phase-folded the light curves with the best period and its double value, then decided which gave a better fit based on a visual inspection.

In our parallel paper about Main Belt asteroid detections we show that the period determination is usually solid if the coverage exceeds five days and the duty cycle is above 60\% (Szabó et al. 2016). Since the conditions are well fulfilled for K2 Trojan asteroids, we could derive reliable solutions for most asteroids. In addition to the automatic processing of period determination all phased light curves were inspected visually, too. We suspect that the occurence of period biases due to unfortunate data distribution is very low. For some objects the light curve we obtained suggested a complex brightness variation. In these cases we tested manually for periods other than the primary one, but finally omitted solutions different from the bimodal light curves. The phased light curves were also binned by 0.02 rotational phase steps, and the full amplitude was accepted as the difference between the minimum and maximum of this binned light curve.

\section{Results}

\subsection{Period and amplitude distributions}

We present the determined periods and amplitudes in Table A.2. Both parameters span over a wide range (which we discuss later in detail), and indicatively, the median value of the measured periods is around $13 \mathrm{~h}$, and the median amplitude is 0.32 . The number of high amplitude asteroids is surprisingly high; all asteroids in our sample exceed 0.1 amplitude, and several asteroids exceed 0.75 amplitude, belonging to more than $2: 1$ asphericity in sky projection. These findings, as well as the overall

\footnotetext{
2 https://github.com/astroML/gatspy/
} 

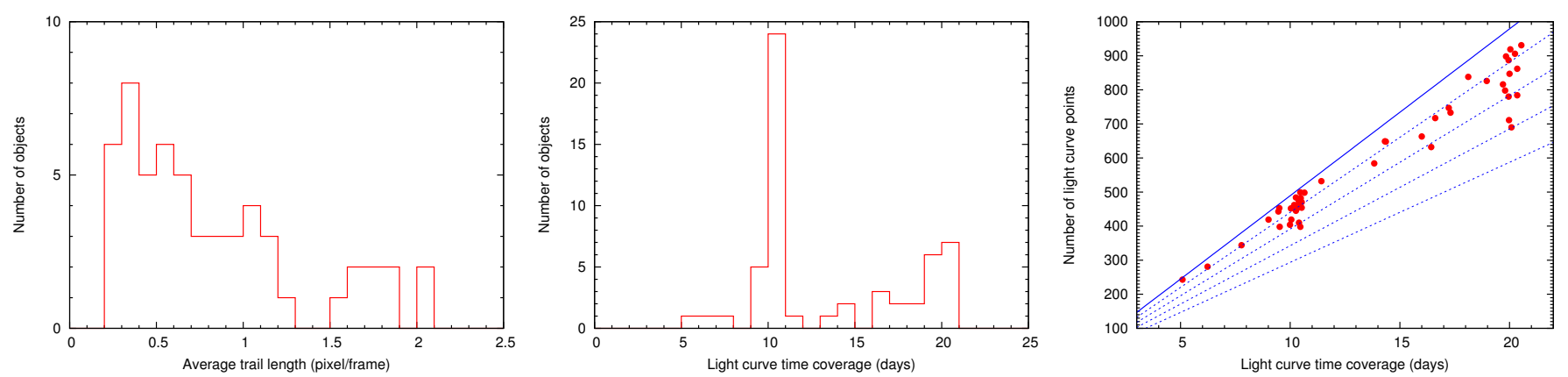

Fig. 3. Statistics related to the light curve acquisition characteristics and the photometric data coverage. See text for further explanation.

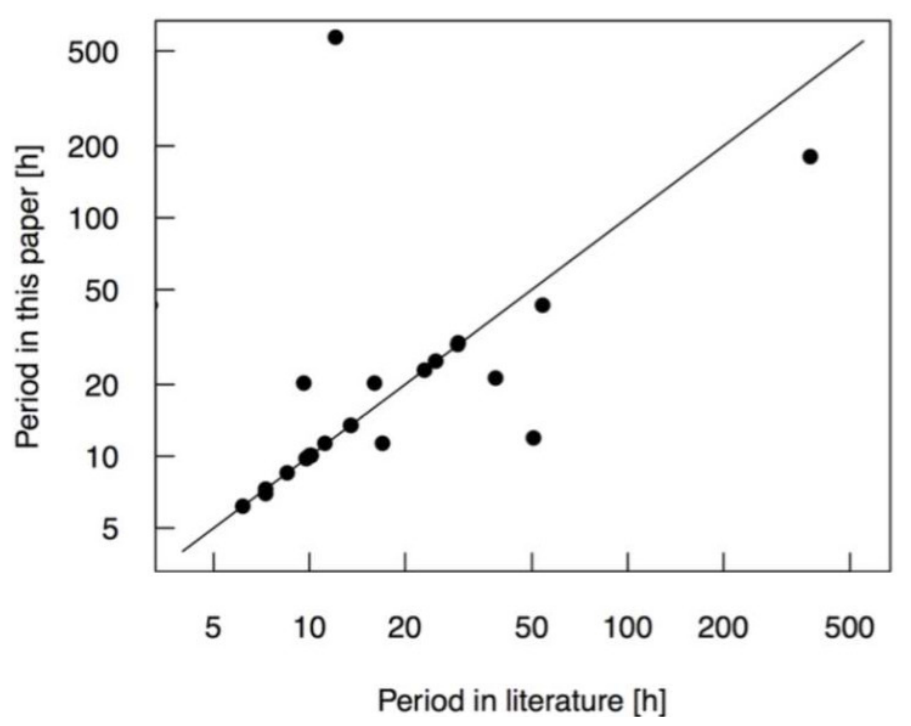

Fig. 4. Comparison of periods in the literature and our determinations.

distribution of our data points, are consistent with the results in previous publications.

Our period determinations are plotted against data from the literature (Molnar et al. 2008; Mottola et al. 2011; French et al. 2015; Waszczak et al. 2015) in Fig. 4. For periods less than ten hours our periods are in perfect coincidence with the previous determinations, while above the $15-18 \mathrm{~h}$ period, we could confirm only a fraction of the previously determined periods. Clearly, the power of the uninterrupted K2 light curves is in its accuracy in the long period range, partly because the measurements are free from daily aliases, and also because the stable observation circumstances do not lead to distracting systematics that mimic rotational light variations.

In Fig. 5 we plot the amplitude-period diagram for Trojans from the literature (Molnar et al. 2008; Mottola et al. 2011; French et al. 2015; Waszczak et al. 2015) compared to our results. It is known that Main Belt families are characterized by specific brightness variation distributions (Szabó \& Kiss 2008). For the Trojan asteroids, we cannot confirm such parameter dependencies.

Our sample shows a significant overabundance above $60 \mathrm{~h}$ periods. Possibly this is due to the limited completeness of previous surveys in the range of very slow rotation rates. Due to the unbiased $\mathrm{K} 2$ observations that last for many days in most cases, we believe that in this range our sample is still balanced and reflects the common occurrence of very slow rotators among Trojan asteroids. This result would be worth comparing to a similar sample in the Main Belt. Interestingly, this is not possible
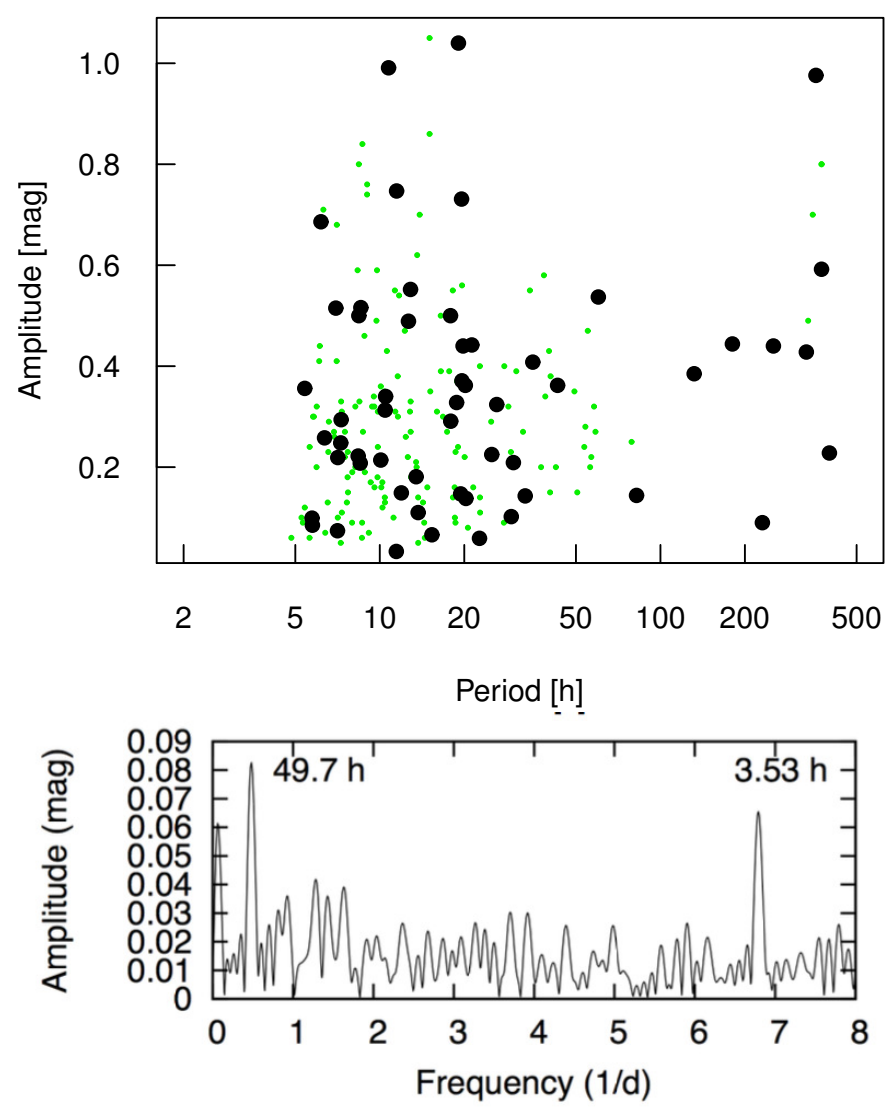

Fig. 5. Upper panel: period-amplitude distribution of Trojan asteroids. Our data are plotted with large black dots, data from the literature is plotted with small green dots. See the text for references and discussion. Lower panel: periodogram of (65227) $2002 \mathrm{ES}_{46}$ with multiple periods.

now, since the already known distributions suffer the same incompleteness as the Trojan asteroids in the very long period range, so we cannot compare our results to the known distributions. Even K2 asteroid surveys are more limited in the very long period range than in the Trojan swarms, simply because Main Belt asteroids move faster and spend much less time in the observed area than Trojan asteroids do.

\subsection{Signs of binarity}

Three light curve features are invoked as alerting signs for a binary asteroid. Leone et al. (1984) defined a light curve amplitude $>0$ m. 9 at least at one viewing geometry, referring to an $a / b$ asphericity $>2.3$. This elongation cannot be explained by a rubble 
pile body in equilibrium, but instead can be explained by a Jacobi ellipsoid stretched along the semi major axis of two rubble piles orbiting each other. Another signal of binarity is the slow rotation of the asteroid, possibly reflecting a tidal synchronization with a modestly far companion. Following Sonnett et al. to put the alerting limit at $3 \times$ the average Trojan period, here we consider a Trojan to rotate unusually slowly if the period exceeds $3 \times$ the average, roughly $40 \mathrm{~h}$. The third signal of possible binarity is the presence of two periods, reflecting the light variation of the main body and the companion which have not been synchronized, or the forced perturbation of the main body because of the presence of the companion.

In our sample, several asteroids exhibited the described diagnostics of binarity. Asteroids 21593, 22056, and 39289 were detected to exceed 0.9 amplitude, and hence became good candidates for binary pile asteroids. 22056 exhibited the slowest rotation rate of $358 \mathrm{~h}$ (almost 15 days), therefore we consider it to be the best and most promising candidate for being a binary Trojan in K2 data.

Very slow rotation only characterized a surprisingly large number of asteroids. Besides the mentioned example of 22056 , we found asteroids $9807(330 \mathrm{~h}), 13331(180 \mathrm{~h}), 23958$ (trend only), 24357 (131 h), 39270 (82 h), 63239 (60 h), 65223 (252 h), $65240(230 \mathrm{~h})$, and $129602(43 \mathrm{~h})$ in the alerting range of rotational rates.

There is one asteroid with observations both in WISE data and K2, (16152) $1999 \mathrm{YN}_{12}$. Sonnett et al. (2015) derived a WISE magnitude range of $0.97 \pm 0.15$, for this asteroid, while here we present a period of $11.47 \pm 0.22$ hours and 0.75 amplitude. Since the amplitude is based on $475 \mathrm{~K} 2$ photometric points, the photometric error is in the order of $0 \mathrm{~m} .01$ in the amplitude. Six years passed between the WISE and K2 measurements, therefore the two amplitudes refer to very different aspect geometry at roughly opposing positions on the asteroid's orbit. Since both amplitudes are unusually high, this body is likely really elongated and has a pole position roughly perpendicular to the plane of Ecliptic. Thus (16152) $1999 \mathrm{YN}_{12}$ passed the amplitude criterion of binarity, and remains a good candidate for further investigation.

Putting these detections together, we get $11 \mathrm{~K} 2$ Trojans with signs of binarity. This means there is $\mathrm{a} \approx 20 \pm 5 \%$ rate of binaries among L4 Trojans, or at least asteroids with strong signs of binarity. This determination is unbiased because of the full light curve coverage and the long observation runs, and is fully compatible with the debiased rate of 14-23\% given by Sonnett et al. from WISE data, and is consistent or slightly exceeds a previous estimate of 6-10\% (Mann et al. 2007) from Earth-based light curves.

\section{3. (65227) $2002 E S_{46}$ with double periods}

We detected multiple periodicities in the case of (65227) 2002 $\mathrm{ES}_{46}$ : the "one-hump" periods are $49.7 \mathrm{~h}$ and $3.53 \mathrm{~h}$, with 0.065 and 0.074 full amplitudes, respectively. The detection of these periods is secure above the noise level of $\approx 0$ m03 -0 m 04 . These periods are not resonant to each other, and since the period ratio is $\approx 14.1$, they seem to have independent origins in processes with very different periods. The amplitudes are tiny, indeed the are the smallest ones in our K2 Trojan sample (Fig. 5 lower panel).

This detection can be considered as another alerting signal for a binary asteroid. Both the periods and the amplitudes can be explained by a binary object with partial tidal locking. Assuming a two-hump light curve solution, the rotation period of one body would be $7.06 \mathrm{~h}$; while the other periodicity of 49.7 (or 99.4) h comes from the orbital motion and possibly, the light variation of the tidally locked rotator.

This interpretation is consistent also with the small amplitudes. Since both periods come from only one body of the system, the presence of the other body significantly suppresses the measured amplitude. Assuming two equally bright components, the full light curve amplitude from each components would be 0 . 16 and 0.12 .

Another possible interpretation would be that the object is in a state of free precession, due to a recent collision. It is known from theoretical physics that an oblate ellipsoid with principal moments of inertia $A=B$ and $C$ will be in a state of free precession if the rotation axis is inclined to the total angular momentum vector, and the ratio of $\omega$ rotation rate and the $\Omega$ precession rate will be $\Omega / \omega=(A-C) / C$, independently of the inclination angle of the rotation vector. If we assume a free precession in the case of (65227) $2002 \mathrm{ES}_{46}$, an ellipsoidal shape and a homogeneous internal composition, we can derive the axis ratios to be $A / C=1.071$. Such a large precession rate can therefore be observed only for an almost spherical body. This numerical result is also consistent with the observed light variation from this object on the order of a few $0 \mathrm{~m} .01$.

However, we consider that the binary scenario is more likely in the case of 65227. We considered that binarity is quite common in our sample. Moreover, in the case of binarity, quite elongated objects can support the observed light variation, since the two sources mutually suppress the amplitudes. The free precession scenario seems to be less likely since the precession rate has stringent implications for the sphericity of the rotating body, and also, the scenario assumes a recent collision in an otherwise relaxed part of the solar system.

\subsection{Comparison of red and less red populations}

We compared the red and less red populations by means of period and amplitude distributions of the member asteroids. For this task, we cross-correlated the K2 asteroids to the SDSS Moving Object Catalog 4 (MOC hereafter), to derive the $t$ color which separates the red and less red members the most (Szabó et al. 2007). In case of multiple measurements of the same asteroid, the measurements were averaged to get more precise colors.

Eleven Trojans with K2 observations were found with an entry in SDSS MOC. Four of them were identified as representing the less red population - (8241) Agrius, 13331, 16152, and 23939 - and seven as belonging to the red population of Trojans - (1749) Telamon (5028) Halaesus, 21599, 22056, 24357,59049 , and 129602 . We added the population membership as a population flag ( $r$ for red, $l r$ for less red) in Table A.2.

No specific pattern has been found for red and/or less red population members. Both groups contain normal rotators around the median value of all $\mathrm{K} 2$ Trojans $(\approx 13 \mathrm{~h}$ period, $\approx 0 \mathrm{~m} .32$ amplitude) and also, both exhibit very slow rotators (e.g., 180 and $358 \mathrm{~h}$ for 13331 and 22056, respectively) and elongated asteroids (e.g., 0.75 and 0.98 for 16152 and 22056, respectively). Because of low counts, we cannot form more detailed conclusions, but we can confidently observe that high amplitude asteroids and slow rotators are not specific to one population only: they are quite common in both populations. 


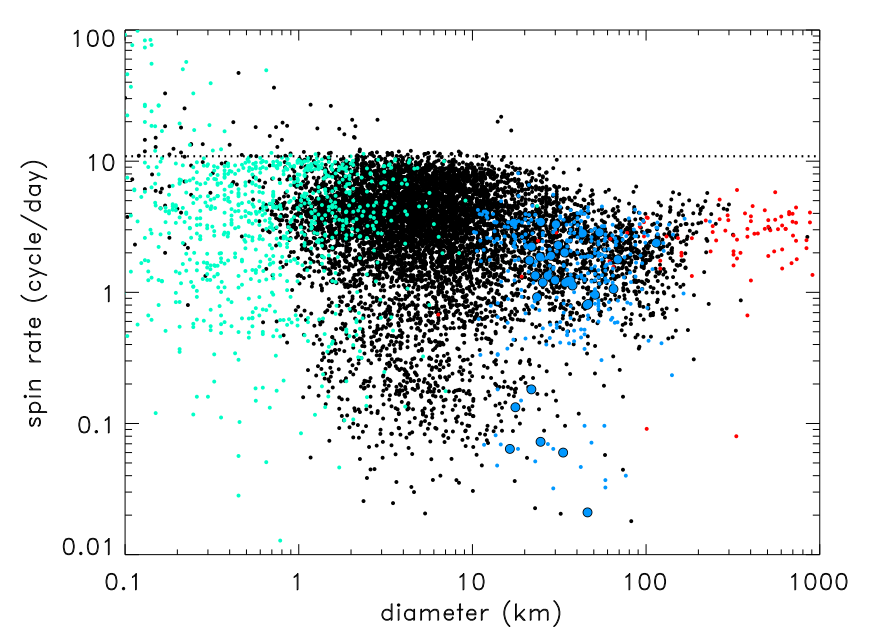

Fig. 6. Diameter versus spin rate of asteroids. Black dots: Main Belt, red dots: trans-Neptunian objects, green dots: near-Earth asteroids, small blue dots: Jovian Trojans. Data is obtained from the Asteroid Light Curve Database (Warner et al. 2009). The Jovian Trojan asteroids with known sizes - as determined by NEOWISE (Grav et al. 2012) - are marked by large, filled blue circles.

\subsection{Period distribution and the spin barrier of Jovian Trojan asteroids}

In Fig. 6 we present the size versus spin rate distribution of small bodies, including Near-Earth and Main Belt asteroids, Jovian Trojans, Centaurs, and Trans-Neptunian objects. The fast rotation of minor planets is limited by the so-called spin barrier: there is a critical rotation period at which a rubble pile asteroid would fly apart due to its centripetal acceleration. For a specific body, this critical period can be estimated as $P_{\mathrm{c}} \approx$ $3.3 \cdot \sqrt{(1+A) / \rho}$, where $A$ is the light curve amplitude, $\rho$ is the density (in $\left[\mathrm{g} \mathrm{cm}^{-3}\right]$ ), and $P_{\mathrm{c}}$ is obtained in hours (Pravec $\&$ Harris 2000). Using this formula the knowledge of the rotation period and the light curve amplitude can provide a lower limit estimate of the body's density. This spin barrier is well established for Main Belt asteroids, the critical rotation period is $\sim 2.2 \mathrm{~h}$ (dashed horizontal line in Fig. 6), resulting in a critical density of $\sim 2.0 \mathrm{~g} \mathrm{~cm}^{-3}$. For Main Belt asteroids, this limit is set by asteroids with diameters of $1-10 \mathrm{~km}$. However, with the Jovian Trojans, we are in the size range of $\sim 10-100 \mathrm{~km}$, that is, we study objects that are an order of magnitude larger than the ones setting the spin barrier for Main Belt asteroids.

Comparison of the spin rate distribution of Jovian Trojans with Main Belt asteroids in the Jovian Trojan size range (Fig. 7) shows a notable lack of Jovian Trojans in the high spin rate range. Based on the data available in their study, French et al. (2015) estimated densities of $\sim 0.5 \mathrm{~g} \mathrm{~cm}^{-3}$ using the fastest rotation periods of $\sim 5 \mathrm{~h}$. This value is different from the critical density obtained for Main Belt asteroids $\left(\sim 2 \mathrm{~g} \mathrm{~cm}^{-3}\right)$ but consistent with the density of cometary nuclei (A'Hearn 2011) as well as that of objects from the trans-Neptunian populations (Brown 2013; Vilenius et al. 2014). Applying the critical density calculation above for our sample, the highest density values we obtain are $\sim 0.5 \mathrm{~g} \mathrm{~cm}^{-3}$ (see Fig. 8), lower than that of Main Belt asteroids in the same size range, but the same as obtained by French et al. (2015). Even in our unbiased sample, despite the fact that rotation periods longer than $\sim 1 \mathrm{~h}$ should have been identified, we were unable to find any Jovian Trojan asteroid with a high $(\sim 2 \mathrm{~h})$ spin rate. This may indicate icy compositions and porous

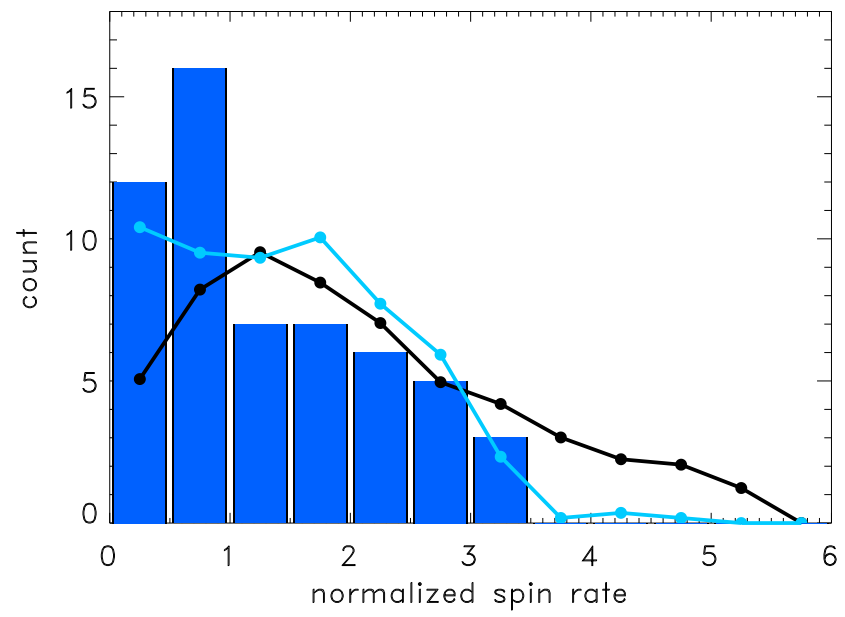

Fig. 7. Distribution of normalized spin rates in our sample (blue bars). We used $\langle f\rangle=1.34$ cycle day $^{-1}$ for normalization. Black and blue curves represent the scaled normalized spin rate distribution of Main Belt and Jovian Trojan asteroids, respectively, as obtained from the Asteroid Light Curve Database (Warner et al. 2009).

interiors for most Jovian Trojans, supporting an outer solar system/Kuiper belt origin, as discussed in Sect. 1.

Jovian Trojans show, in general, an excess in the number of slow rotators when compared with Main Belt asteroids (blue and black curves in Fig. 7). In our sample this is even more expressed, as there is a significant number of asteroids with periods $P>50 \mathrm{~h}, \sim 20 \%$ of the objects observed by $\mathrm{K} 2$ in this work. Very long rotation periods may be an indication of binarity, as is the case, for example, for Jovian Trojan (617) Patroclus where the $>100 \mathrm{~h}$ rotation period is explained by tidal breaking (Mueller et al. 2010). Slow rotation of small $(<30 \mathrm{~km})$ and low density $\left(<1 \mathrm{~g} \mathrm{~cm}^{-3}\right)$ objects may have also been set by the YORP effect (French et al. 2015). Among our slow rotators, there are objects that fall into this susceptible size range according to the sizes derived by Grav et al. (2012) based on NEOWISE observations.

\subsection{Light curve amplitude distribution}

In Fig. 9 we plotted the amplitude distribution of our Trojan asteroid sample (black bars) and compared it with the amplitude distribution obtained by Binzel \& Sauter (2011) for a somewhat smaller sample. The magnitude limit of the detectability of periodic light curve variation for our sample is estimated to be $\Delta m_{\min } \approx 0.02$ that corresponds to a $a / b$ axis ratio of $(a / b)>$ $10^{0.4 \cdot \Delta m_{\min }}=1.02$, if only shape effects are taken into account. We note again that light curve variations were detected for all asteroids in our sample; therefore our sample can be considered to be an unbiased sample, rather than the sample by Binzel \& Sauter (2011), where the targets were mostly Jovian Trojan asteroids for which the existence of detectable brightness variations were previously known. A selection bias for the Binzel \& Sauter (2011) sample can also be inferred from the fact that a Kolmogorov-Smirnov test that compares the two cases gives a probability of only $\sim 7 \%$ that the two samples are drawn from the same distribution.

Our light curves are certainly affected by the spin axis orientations. To test the impact of the geometry on the amplitude statistics, we have corrected the original amplitudes following the method given by Binzel, that is, choosing the largest value if multiple amplitudes are available in the Asteroid Light Curve 
Gy. M. Szabó et al.: K2 photometry of Jupiter Trojan asteroids
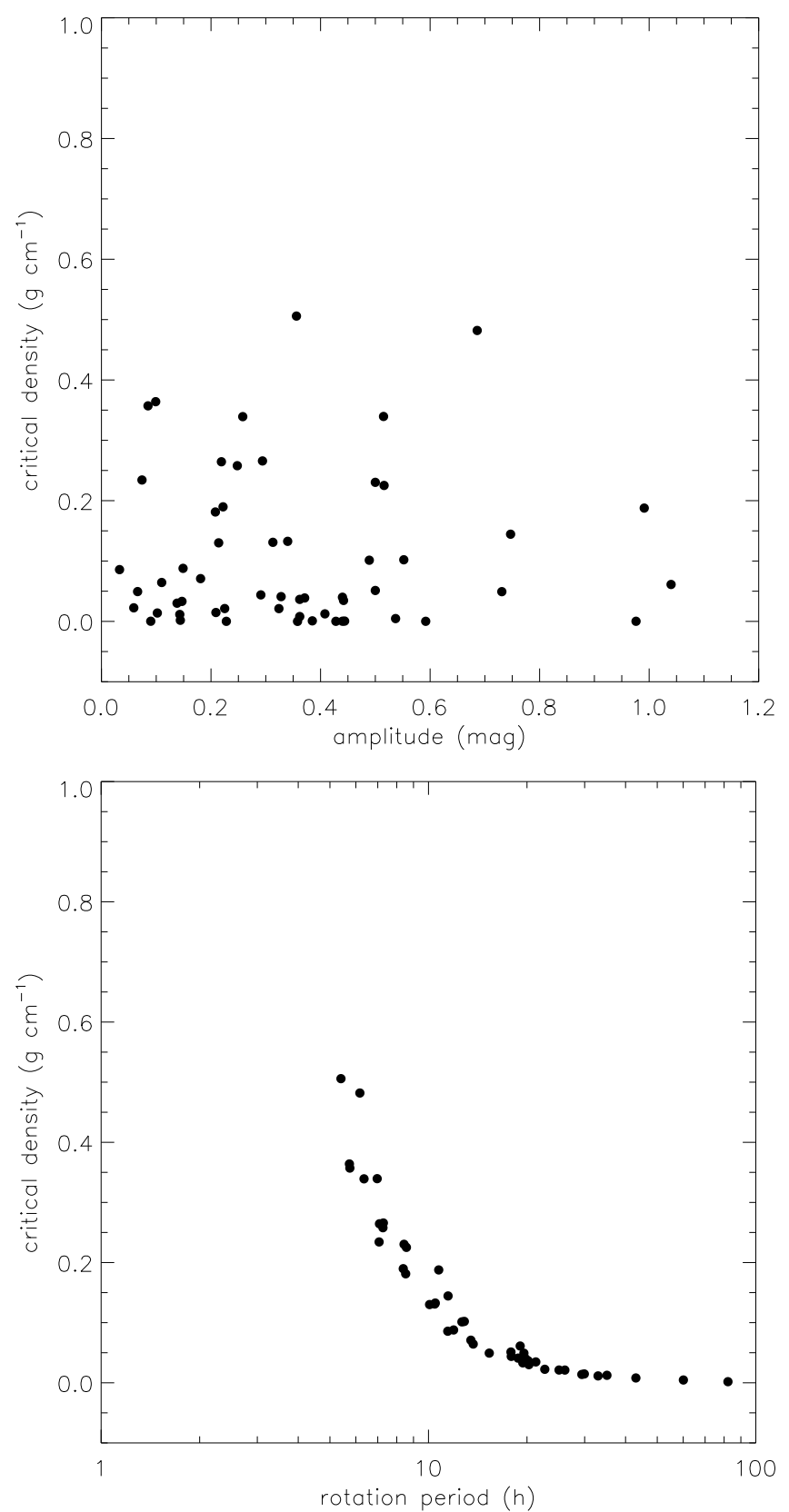

Fig. 8. Critical densities obtained for our sample, as a function of light curve amplitude (upper panel) and rotation period (lower panel).

Database (Warner et al. 2009), and applying a correction assuming $\vartheta=60^{\circ}$ aspect angle in the case of single amplitudes.

The higher frequency of large amplitudes in our sample with respect to Main Belt asteroids is even more pronounced than in the sample of Binzel \& Sauter (2011, see Fig. 20 in their paper). Binzel \& Sauter (2011) interpreted this as a higher number of elongated objects in the Trojan population, however, it is still unknown how the collisional history or other evolutionary effects can explain this deviation.

\section{Summary}

In this paper we investigated the Trojan L4 asteroid detections by $\mathrm{K} 2$ and got the following conclusions:

- The K2 sample shows a significant fraction $(\approx 20 \%)$ of very slow $(P>50 \mathrm{~h})$ rotation periods. The $\mathrm{K} 2$ sample is still

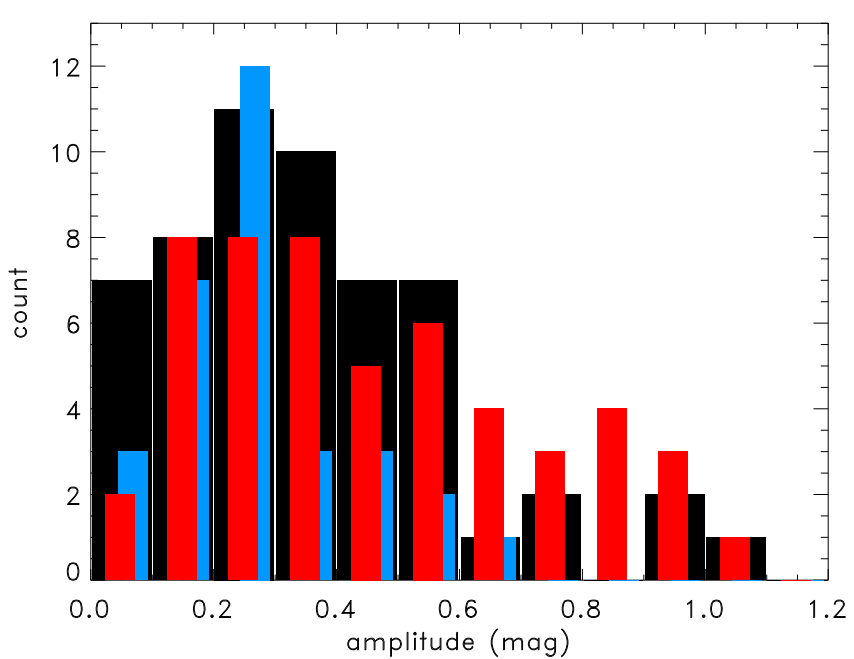

Fig. 9. Light curve amplitude distribution of Jovian Trojan asteroids. Black bars represent our original, uncorrected sample. The red and blue bars correspond to the bias-corrected amplitudes of our sample and that in Binzel \& Sauter (2011).

unbiased in this period range, therefore this observation reflects the actual occurrence of very slow rotators.

- The K2 sample shows a significant overabundance of large amplitude asteroids. Three of 56 asteroids exceeded the 0.9 amplitude, and five $(\approx 10 \%)$ Trojans exceed 0.714 , belonging to $a / c>2$ asphericity.

- In the case of (65227) $2002 E S_{46}$ we detected double periodicity, 49.7 and $3.53 \mathrm{~h}$ and 0.065 and 0.074 full amplitudes, respectively. This is by far the largest asteroid known with a double period.

- The excess of large amplitude asteroids, the very slow rotators among Trojans, and the presence of double periods can all be explained by a high rate of binary asteroids in the L4 cloud. We estimated the occurrence of binarity to be between $20-25 \%$, in agreement with previous estimates.

- Red and less red populations were found to be identical for light variation properties. The similarity applies for both amplitude and period distributions, and also, the presence of very slow rotators and high amplitude asteroids.

- We detected a notable lack of fast rotators among Trojans. We interpreted this as the effect of a density barrier, and estimated the upper limit of the density of $0.5 \mathrm{~g} / \mathrm{cm}^{3}$ in the $\mathrm{K} 2$ sample, in agreement with previous estimates.

- We derived the amplitude distribution of K2 Trojan asteroids and debiased assuming random spin orientation. Both the observed and unbiased distributions differ from the results of Binzel \& Sauter (2011) since K2 observed significantly more asteroids in the high amplitude wing of the distribution. How the collisional history or other evolutionary effects led to the excess of elongated bodies in the Trojan cloud still needs further investigation.

Acknowledgements. Funding for the Kepler and K2 missions is provided by the NASA Science Mission directorate. The authors acknowledge the Kepler team for their extra efforts to allocate special pixel masks to track moving targets. Targets were selected and proposed by Erin Ryan (GO6025). This work has been supported by the Lendület Programme of the Hungarian Academy of Sciences (LP2012-31, LP2014-17), by the OTKA K-109276 and K-104607, the GINOP2.3.2-15-2016-00003 grant and NKFIH K-115709 and PD-116175 grants of the Hungarian National Research, Development and Innovation Office, a TéT14FR-1-2015-0012 grant, and by the City of Szombathely under agreement No. $67 / 177-21 / 2016$. The research leading to these results has received funding from 
the European Communitys Seventh Framework Programme (FP7/2007-2013) under grant agreement No. 312844 (SPACEINN), the ESA PECS Contract Nos. 4000110889/14/NL/NDe and 4000109997/13/NL/KML, the European Unions Horizon 2020 Research and Innovation Programme, Grant Agreement no 687378. L.M. was supported by the János Bolyai Research Scholarship of the Hungarian Academy of Sciences. All of the data presented in this paper were obtained from the Mikulski Archive for Space Telescopes (MAST). STScI is operated by the Association of Universities for Research in Astronomy, Inc., under NASA contract NAS5-26555. Support for MAST for non-HST data is provided by the NASA Office of Space Science via grant NNX13AC07G and by other grants and contracts.

\section{References}

A'Hearn, M. F. 2011, ARA\&A, 49, 281

Binzel, R. P., \& Sauter, L. M. 1992, Icarus, 95, 222

Brown, M. E. 2013, ApJ, 778, L34

Dahlgren, M. 1998, A\&A, 336, 1056

Dell'Oro, A., Marzari, F., Paolicchi, P., \& Vanzani, V. 2001, A\&A, 366, 1053

Emery, J. P., Marzari, F., Morbidelli, A., French, L. M., \& Grav, T. 2016, in Asteroids IV, eds. P. Michel, F. E. DeMeo, \& W. F. Bottke (Tucson: University of Arizona Press)

French, L. M., Stephens, R. D., Coley, D., Wasserman, L. H., \& Sieben, J. 2015, Icarus, 254, 1

Grav, T., Mainzer, A. K., Bauer, J., et al. 2011, ApJ, 742, 40

Grav, T., Mainzer, A. K., Bauer, J. M., Masiero, J. R., \& Nugent, C. R. 2012 , ApJ, 759, 49

Gomes, R., Levison, H. F., Tsiganis, K., \& Morbidelli, A. 2005, Nature, 435, 466 Howell, S. B., Sobeck, C., Haas, M., et al. 2014, PASP, 126, 398 Kiss, Cs., Pál, A., Farkas-Takács, A. I., et al. 2016, MNRAS, 457, 2908

Lacerda, P., Fornasier, S., Lellouch, E., et al. 2014, ApJ, 793, L2

Lenz, P., \& Breger, M. 2005, Commun. Asteroseismol., 146, 53

Leone, G., Paolicchi, P., Farinella, P., \& Zappalá, V. 1984, A\&A, 140, 265
Levison, H. F., Morbidelli, A., Tsiganis, K., Nesvorný, D., \& Gomes, R. 2011, AJ, 142, 152

Mann, R. K., Jewitt, D., \& Lacerda, P. 2007, AJ, 134, 1133

Merline, W. J., Weidenschilling, S. J., Durda, D. D., et al. 2002, Asteroids do have satellites, in Asteroids III, eds. W. F. Bottke Jr., A. Cellino, P. Paolicchi, \& R. P. Binzel (Tucson: University of Arizona Press), 289

Molnar, L. A., Haegert, M. J., \& Hoogeboom, K. M. 2008, Minor Planet Bull., 35,82

Morbidelli, A., Levison, H. F., Tsiganis, K., \& Gomes, R. 2005, Nature, 435, 462

Mottola, S., Di Martino, M., Erikson, A., et al. 2011, AJ, 141, 170

Mueller, M., Marchis, F., Emery, J. P., et al. 2010, Icarus, 205, 505

Nesvorný, D., Vokrouhlický, D., \& Morbidelli, A. 2013, ApJ, 768, 45

Noll, K. S., Grundy, W. M., Chiang, E. I., Margot, J.-L., \& Kern, S. D. 2008, in The solar system Beyond Neptune, eds. A. Barucci, H. Boehnhardt,

D. Cruikshank, \& A. Morbidelli (Tucson: University of Arizona Press), 345

Pál, A. 2012, MNRAS, 421, 1825

Pál, A., Szabó, R., Szabó, Gy, M., et al. 2015, ApJ, 804, L45

Pál, A., Kiss, Cs., Müller, Th. G., et al. 2016, AJ, 151, 117

Peixinho, N., Delsanti, A., Guilbert-Lepoutre, A., Gafeira, R., \& Lacerda, P. 2012, A\&A, 546, A86

Pravec, P., \& Harris, A. W. 2000, Icarus, 148, 12

Roig, F., Ribeiro, A. O., \& Gil-Hutton, R. 2008, A\&A, 483, 911

Sonnett, S., Mainzer, A., Grav, T., Masiero, J., \& Bauer, J. 2015, ApJ, 799, 191

Szabó, G. M., Ivezić, Ž., Jurić, M., \& Lupton, R. 2007, MNRAS, 377, 1393

Szabó, R., Sárneczky, K., Szabó, Gy, M., et al. 2015, AJ, 149, 112

Szabó, R., Pál, A., Sárneczky, K., et al. 2016, A\&A, 596, A40

Tsiganis, K., Gomes, R., Morbidelli, A., \& Levison, H. F. 2005, Nature, 435, 459

Vilenius, E., Kiss, Cs., Müller, Th. G., et al. 2014, A\&A, 564, A35

Warner, B. D., Harris, A. W., \& Pravec, P. 2009, Icarus, 202, 134

Waszczak, A., Chang, C.-K., Ofek, E. O., et al. 2015, AJ, 150, 75

Wong, I., \& Brown, M. E. 2015, AJ, 150, 147

Wong, I., \& Brown, M. E. 2016, AJ, 152, 90

Wong, I., Brown, M. E., \& Emery, J. P. 2014, AJ, 148, 112 
Gy. M. Szabó et al.: K2 photometry of Jupiter Trojan asteroids

\section{Appendix A: Tables and additional figure}

Table A.1. Summary of the observational characteristics of the 56 Trojans observed by K2 during Campaign 6 .

\begin{tabular}{|c|c|c|c|c|}
\hline (MPC number) & $\begin{array}{l}{ }^{b} \text { Trail } \\
\text { length } \\
\text { (pixel) }\end{array}$ & $\begin{array}{r}{ }^{c} \text { Trail } \\
\text { var. } \\
\text { (pixel) }\end{array}$ & $\begin{array}{r}{ }^{d} \mathrm{LC} \\
\text { points }\end{array}$ & $\begin{array}{r}{ }^{e} \text { Time } \\
\text { cov. } \\
\text { (days) }\end{array}$ \\
\hline (1143) Odysseus & 2.05 & 0.11 & 375 & 9.52 \\
\hline (1749) Telamon & 0.36 & 0.11 & 629 & 20.09 \\
\hline (3801) Thrasymedes & 1.74 & 0.11 & 367 & 10.40 \\
\hline (4035) $1986 \mathrm{WD}$ & 0.84 & 0.10 & 442 & 10.42 \\
\hline (4057) Demophon & 0.26 & 0.14 & 871 & 20.25 \\
\hline (4138) Kalchas & 0.66 & 0.15 & 489 & 10.46 \\
\hline (5028) Halaesus & 0.84 & 0.09 & 466 & 10.48 \\
\hline (5123) 1989 BL & 1.11 & 0.13 & 461 & 10.46 \\
\hline (5244) Amphilochos & 0.32 & 0.10 & 808 & 18.12 \\
\hline (5436) Eumelos & 0.79 & 0.12 & 444 & 10.40 \\
\hline (5652) Amphimachus & 0.31 & 0.12 & 747 & 19.96 \\
\hline (8241) Agrius & 1.84 & 0.13 & 461 & 10.38 \\
\hline (9807) $1997 \mathrm{SJ}_{4}$ & 0.59 & 0.13 & 383 & 10.46 \\
\hline (10989) Dolios & 0.25 & 0.14 & 860 & 19.84 \\
\hline (11251) Icarion & 0.37 & 0.10 & 620 & 14.32 \\
\hline (12238) Actor & 1.17 & 0.10 & 488 & 10.65 \\
\hline (12974) Halitherses & 2.02 & 0.11 & 456 & 10.40 \\
\hline (13184) Augeias & 1.01 & 0.14 & 435 & 10.36 \\
\hline (13185) Agasthenes & 0.76 & 0.12 & 406 & 9.01 \\
\hline (13331) $1998 \mathrm{SU}_{52}$ & 1.13 & 0.14 & 432 & 10.40 \\
\hline (13366) $1998 \mathrm{US}_{24}$ & 1.55 & 0.12 & 387 & 10.05 \\
\hline (13372) $1998 \mathrm{VU}_{6}$ & 1.71 & 0.11 & 430 & 9.46 \\
\hline (13379) $1998 \mathrm{WX}_{9}$ & 0.67 & 0.13 & 449 & 10.52 \\
\hline (14690) $2000 \mathrm{AR}_{25}$ & 0.27 & 0.14 & 894 & 120.54 \\
\hline (14791) Atreus & 0.22 & 0.14 & 514 & 11.42 \\
\hline (15529) $2000 \mathrm{AA}_{80}$ & 1.86 & 0.13 & 432 & 10.20 \\
\hline (16152) $1999 \mathrm{YN}_{12}$ & 1.00 & 0.13 & 475 & 10.26 \\
\hline (21593) $1998 \mathrm{VL}_{27}$ & 0.30 & 0.04 & 235 & 5.09 \\
\hline (21599) $1998 \mathrm{WA}_{15}$ & 0.42 & 0.08 & 888 & 20.04 \\
\hline (22056) $2000 \mathrm{AU}_{31}$ & 0.30 & 0.11 & 833 & 20.35 \\
\hline (23939) $1998 \mathrm{TV}_{33}$ & 1.65 & 0.12 & 453 & 10.42 \\
\hline (23947) $1998 \mathrm{UH}_{16}$ & 1.61 & 0.14 & 437 & 10.16 \\
\hline (23958) $1998 \mathrm{VD}_{30}$ & 0.90 & 0.04 & 767 & 19.80 \\
\hline (24357) $2000 \mathrm{AC}_{115}$ & 0.54 & 0.06 & 812 & 20.00 \\
\hline (24534) $2001 \mathrm{CX}_{27}$ & 1.04 & 0.13 & 446 & 9.50 \\
\hline (24537) $2001 \mathrm{CB}_{35}$ & 0.34 & 0.06 & 641 & 16.00 \\
\hline (35363) $1997 \mathrm{TV}_{28}$ & 0.63 & 0.09 & 335 & 7.78 \\
\hline (39270) $2001 \mathrm{AH}_{11}$ & 0.42 & 0.09 & 781 & 19.70 \\
\hline (38574) $1999 \mathrm{WS}_{4}$ & 0.53 & 0.06 & 682 & 16.61 \\
\hline (39286) $2001 \mathrm{CX}_{6}$ & 0.69 & 0.08 & 273 & 6.23 \\
\hline (39289) $2001 \mathrm{CT}_{28}$ & 0.25 & 0.12 & 707 & 17.31 \\
\hline (57041) $2001 \mathrm{EN}_{12}$ & 0.58 & 0.06 & 682 & 19.98 \\
\hline (58480) 1996 RJ 33 & 0.75 & 0.14 & 451 & 10.44 \\
\hline (59049) $1998 \mathrm{TC}_{31}$ & 0.90 & 0.04 & 851 & 19.96 \\
\hline (63239) $2001 \mathrm{BD}_{25}$ & 0.49 & 0.08 & 558 & 13.83 \\
\hline (65210) Stichius & 0.42 & 0.09 & 719 & 17.23 \\
\hline (65223) $2002 \mathrm{EU}_{34}$ & 0.29 & 0.12 & 620 & 14.36 \\
\hline (65227) $2002 \mathrm{ES}_{46}$ & 0.37 & 0.15 & 440 & 10.18 \\
\hline (65240) $2002 \mathrm{EU}_{106}$ & 0.53 & 0.06 & 596 & 16.43 \\
\hline (65257) $2002 \mathrm{FU}_{36}$ & 0.92 & 0.13 & 427 & 10.26 \\
\hline (83984) $2002 \mathrm{GL}_{77}$ & 0.68 & 0.12 & 387 & 9.99 \\
\hline (88227) $2001 \mathrm{BU}_{42}$ & 0.59 & 0.06 & 744 & 20.35 \\
\hline (88241) $2001 \mathrm{CD}_{23}$ & 0.40 & 0.09 & 793 & 18.96 \\
\hline (129602) $1997 \mathrm{WA}_{12}$ & 1.04 & 0.11 & 452 & 10.40 \\
\hline (228102) $2008 \mathrm{SY}_{172}$ & 0.82 & 0.05 & 435 & 10.52 \\
\hline
\end{tabular}

Notes. The columns are the following: ${ }^{(a)}$ MPC designation of the asteroid; ${ }^{(b)}$ the average apparent trail length (in pixels) of the object on the $\mathrm{K} 2 \mathrm{CCD}$ frames; ${ }^{(c)}$ the variations of the apparent trail length throughout the observations; ${ }^{(d)}$ the number of light curve points used in the further analysis; and ${ }^{(e)}$ the total time coverage of the light curves in days.
Table A.2. Rotational properties of the observed Trojans.

\begin{tabular}{|c|c|c|c|c|}
\hline $\begin{array}{l}{ }^{a} \text { Object } \\
\text { number }\end{array}$ & $\begin{array}{r}\text { Period } \\
\text { (h) }\end{array}$ & $\begin{array}{r}\text { Period } \\
\text { error } \\
(\mathrm{h})\end{array}$ & $\begin{array}{r}\text { Amplitude } \\
\text { (mag) }\end{array}$ & Population \\
\hline 1143 & 10.079 & 0.194 & 0.214 & \\
\hline 1749 & 22.662 & 0.193 & 0.059 & $\mathrm{r}$ \\
\hline 3801 & 20.270 & 0.672 & 0.138 & \\
\hline 4035 & 13.475 & 0.156 & 0.181 & \\
\hline 4057 & 29.925 & 0.765 & 0.209 & \\
\hline 4138 & 29.411 & 2.001 & 0.102 & \\
\hline 5028 & 25.052 & 1.091 & 0.225 & $\mathrm{r}$ \\
\hline 5123 & 19.800 & 0.140 & 0.44 & \\
\hline 5244 & 19.566 & 0.088 & 0.731 & \\
\hline 5436 & 21.276 & 0.315 & 0.442 & \\
\hline 5652 & 8.374 & 0.112 & 0.222 & \\
\hline 8241 & 17.902 & 0.230 & 0.291 & $\operatorname{lr}$ \\
\hline 9807 & 331.034 & 117.56 & 0.428 & \\
\hline 10989 & 26.101 & 0.286 & 0.324 & \\
\hline 11251 & 10.448 & 0.068 & 0.313 & \\
\hline 12238 & 7.281 & 0.041 & 0.294 & \\
\hline 12974 & 6.971 & 0.030 & 0.515 & \\
\hline 13184 & 11.934 & 0.119 & 0.149 & \\
\hline 13185 & 11.453 & 0.124 & 0.033 & \\
\hline 13331 & 180.451 & 31.938 & 0.444 & $\operatorname{lr}$ \\
\hline 13366 & 400 & 105.26 & 0.228 & \\
\hline 13372 & 20.176 & 0.327 & 0.362 & \\
\hline 13379 & 13.698 & 0.138 & 0.11 & \\
\hline 14690 & 8.519 & 0.030 & 0.208 & \\
\hline 14791 & 19.615 & 0.259 & 0.371 & \\
\hline 15529 & 375 & 91.019 & 0.592 & \\
\hline 16152 & 11.477 & 0.223 & 0.747 & $\operatorname{lr}$ \\
\hline 21593 & 10.747 & 0.196 & 0.991 & \\
\hline 21599 & 12.651 & 0.121 & 0.489 & $\mathrm{r}$ \\
\hline 22056 & 358.208 & 16.791 & 0.976 & $\mathrm{r}$ \\
\hline 23939 & 12.868 & 0.104 & 0.552 & $\operatorname{lr}$ \\
\hline 23947 & 15.335 & 0.506 & 0.066 & \\
\hline 23958 & 1142.85 & 154.44 & 0.358 & \\
\hline 24357 & 131.868 & 0 & 0.385 & $\mathrm{r}$ \\
\hline 24534 & 19.417 & 0.368 & 0.147 & \\
\hline 24537 & 10.489 & 0.064 & 0.34 & \\
\hline 35363 & 18.779 & 0.374 & 0.328 & \\
\hline 38574 & 7.085 & 0.084 & 0.219 & \\
\hline 39270 & 82.191 & 4.450 & 0.144 & \\
\hline 39286 & 8.421 & 0.065 & 0.5 & \\
\hline 39289 & 19.062 & 0.160 & 1.04 & \\
\hline 57041 & 8.562 & 0.027 & 0.516 & \\
\hline 58480 & 32.967 & 1.815 & 0.143 & \\
\hline 59049 & 6.172 & 0.012 & 0.686 & $\mathrm{r}$ \\
\hline 63239 & 60.075 & 1.940 & 0.537 & \\
\hline 65210 & 35.087 & 0.626 & 0.408 & \\
\hline 65223 & 252.631 & 67.368 & 0.44 & \\
\hline $65227^{a}$ & 3.53 & 0.025 & 0.074 & \\
\hline $65227^{a}$ & 49.7 & 0.025 & 0.065 & \\
\hline 65240 & 230.769 & 54.945 & 0.09 & \\
\hline 65257 & 17.863 & 0.235 & 0.5 & \\
\hline 83984 & 5.752 & 0.037 & 0.085 & \\
\hline 88227 & 6.355 & 0.018 & 0.258 & \\
\hline 88241 & 5.734 & 0.013 & 0.099 & \\
\hline 353363 & 5.403 & 0.020 & 0.356 & \\
\hline 129602 & 43.010 & 1.188 & 0.362 & $\mathrm{r}$ \\
\hline 228102 & 7.259 & 0.039 & 0.248 & \\
\hline
\end{tabular}

Notes. ${ }^{(a)}$ Asteroid 65227 showed a double periodicity (see text). 
A\&A 599, A44 (2017)

1143

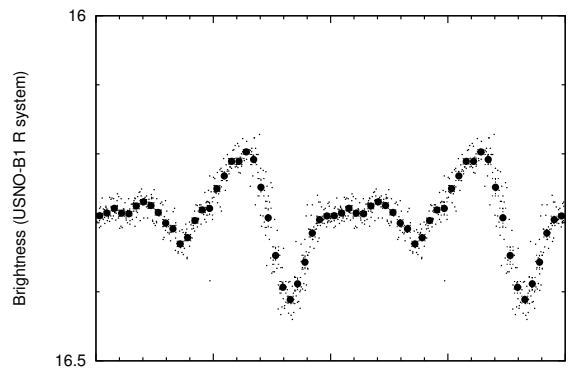

4035

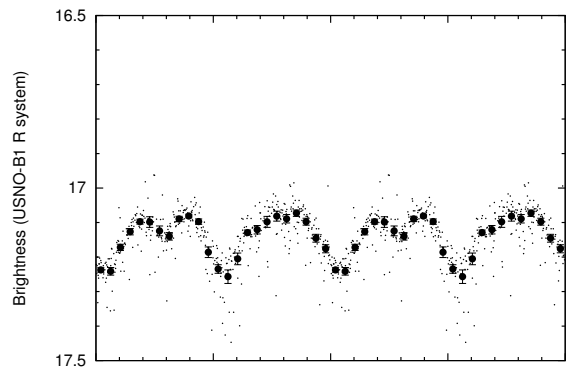

5028

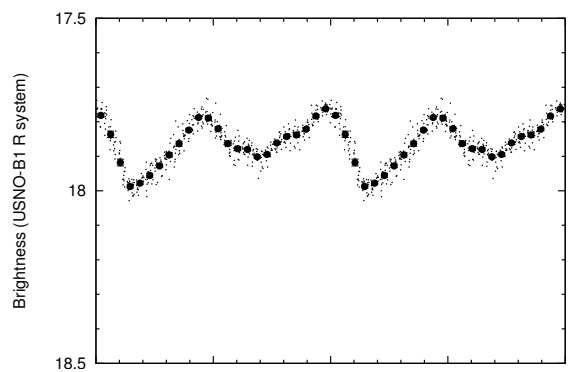

5436

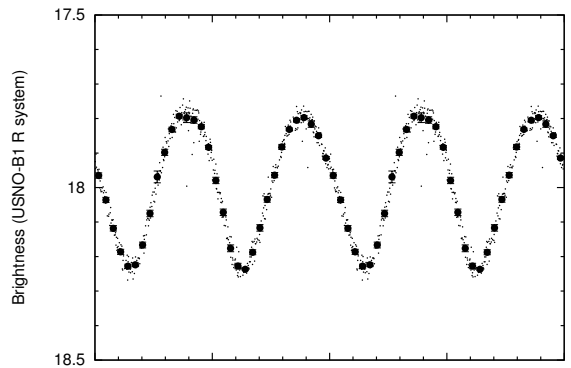

9807

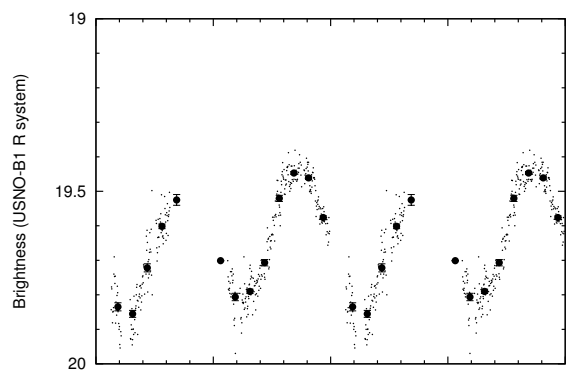

1749

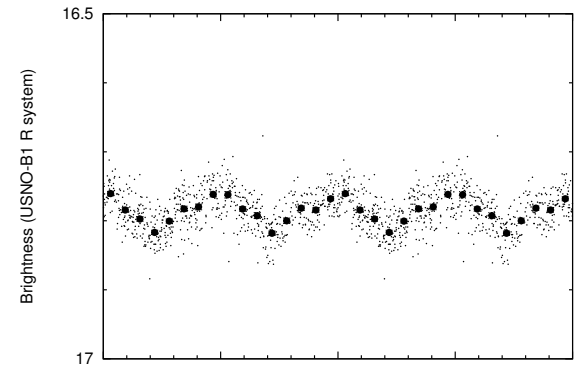

4057

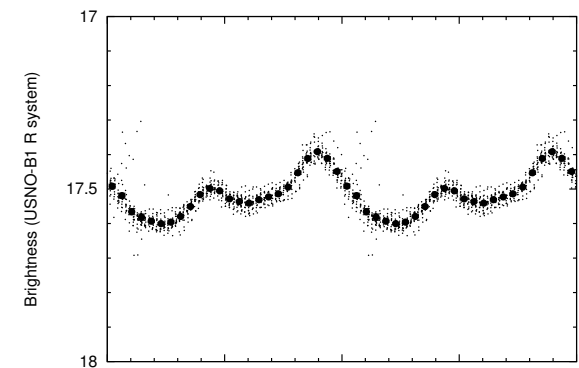

5123

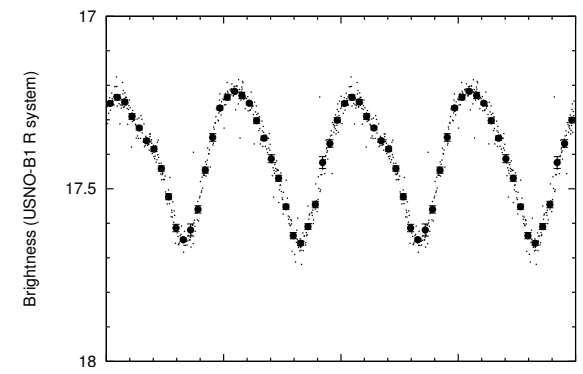

5652

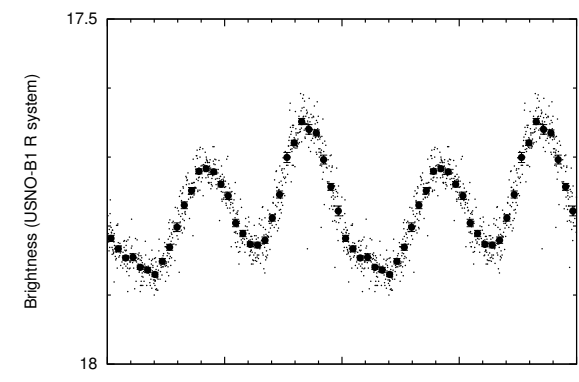

10989

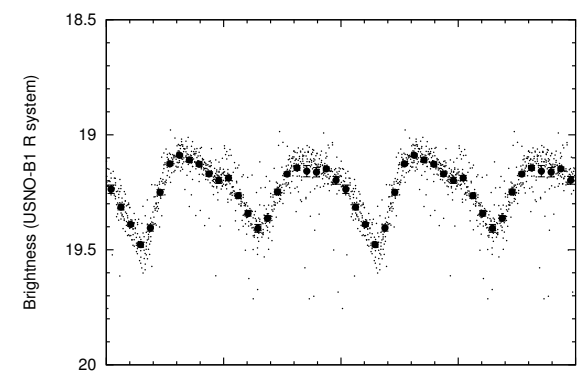

3801

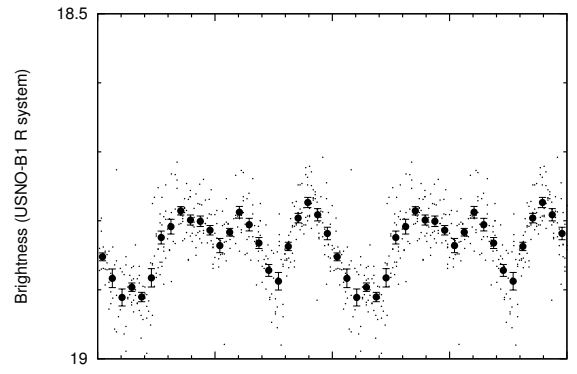

4138

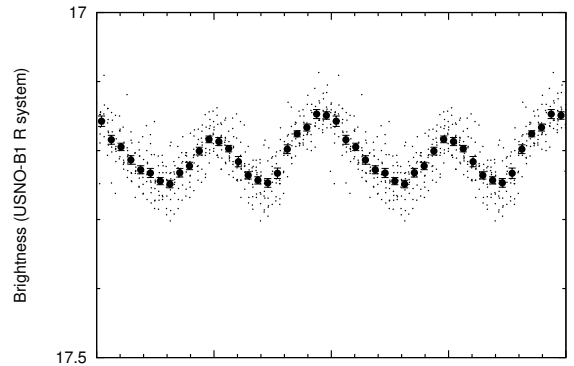

5244

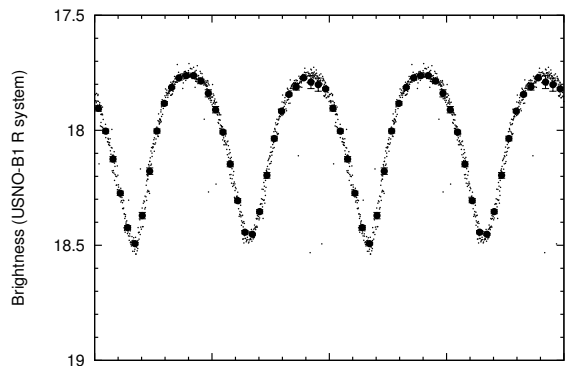

8241

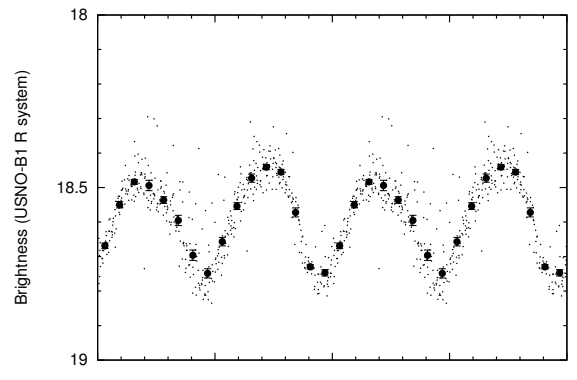

11251

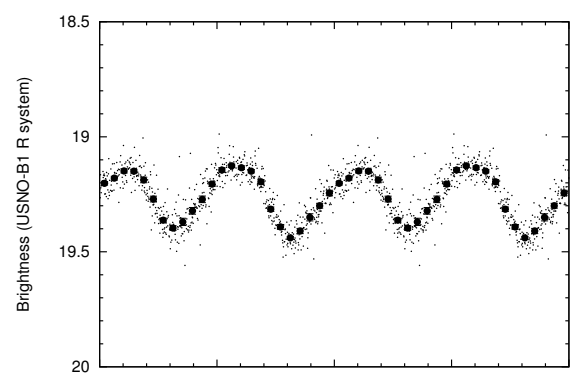

Fig. A.1. Phased light curves of the Trojan asteroids in K2 Field 6. 
Gy. M. Szabó et al.: K2 photometry of Jupiter Trojan asteroids

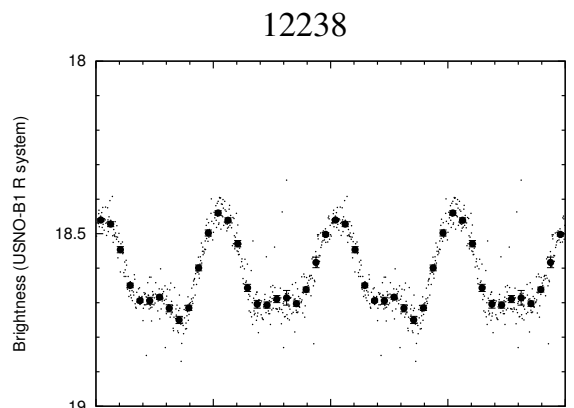

13185

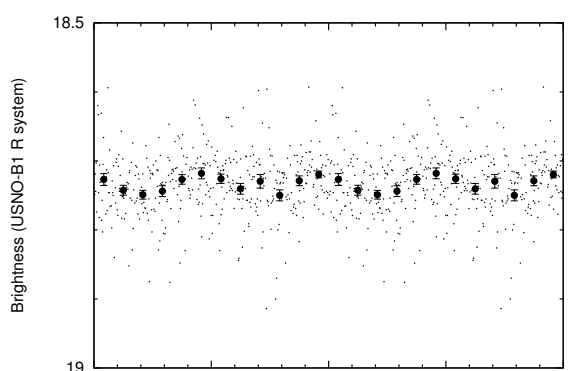

13372

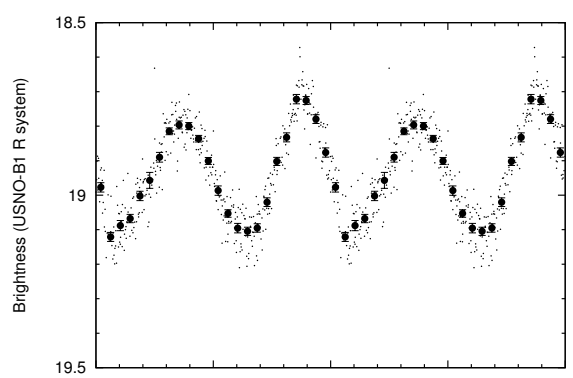

14791

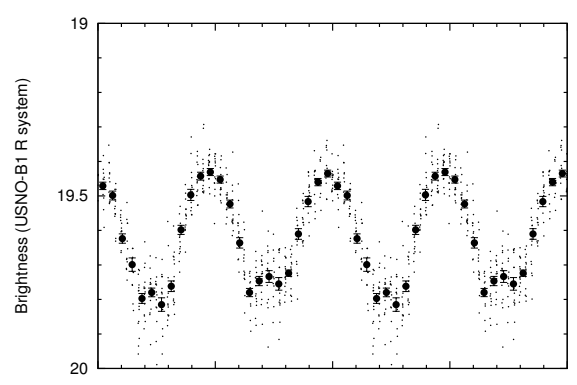

21593

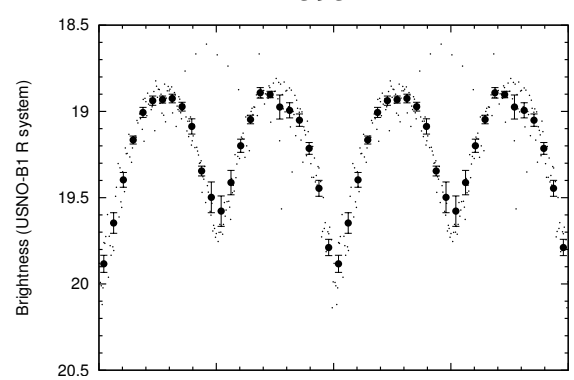

Fig. A.1. continued.
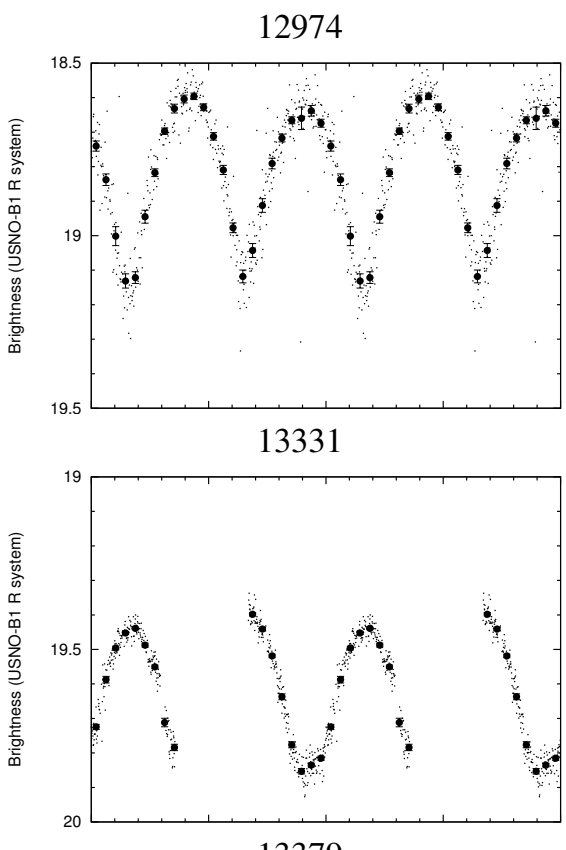

13379

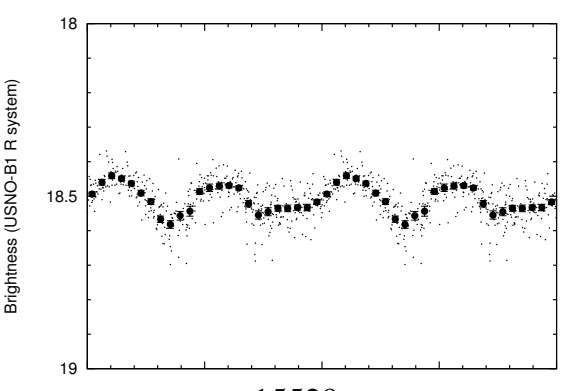

15529

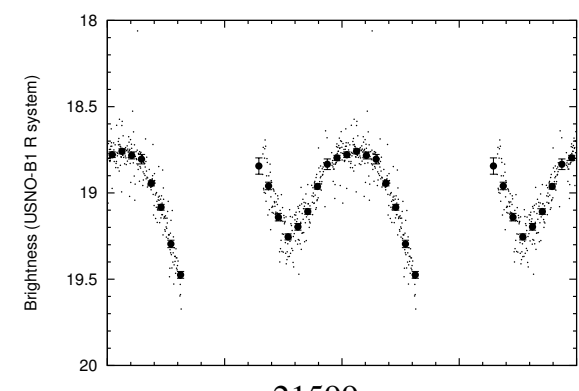

21599

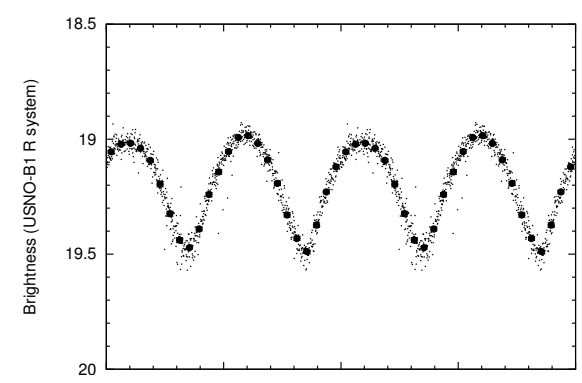

13184
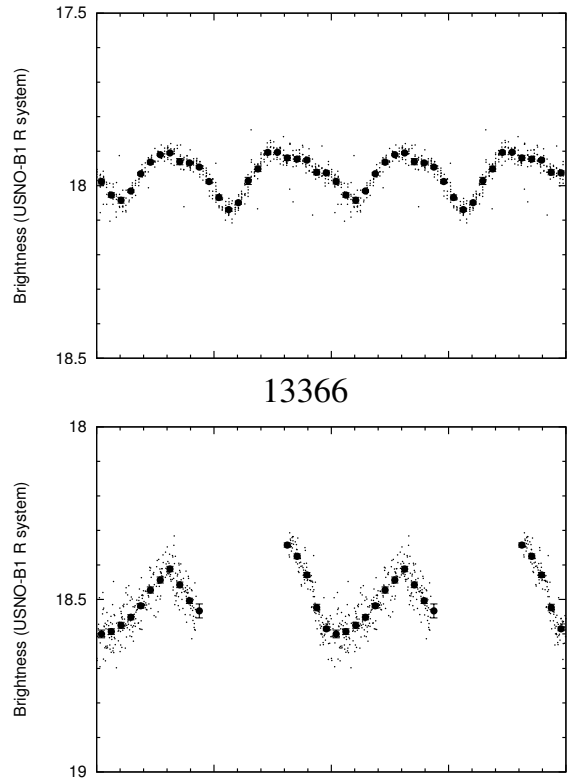

14690

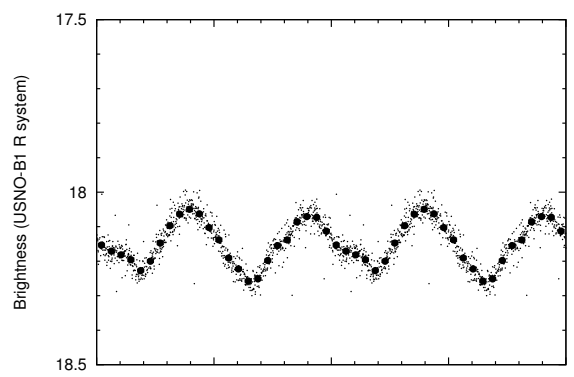

16152

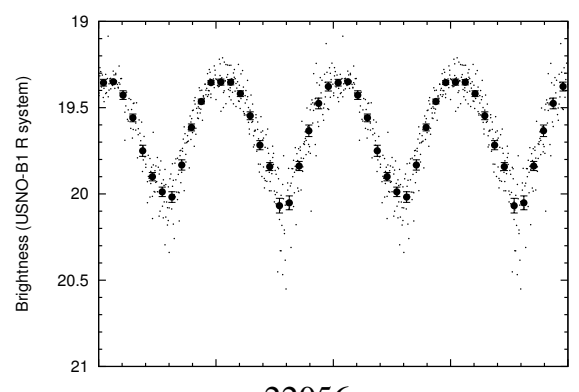

22056

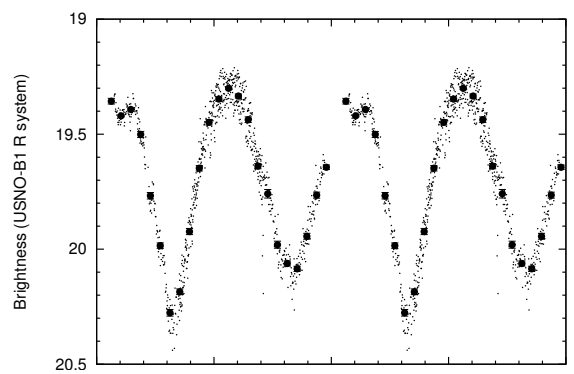



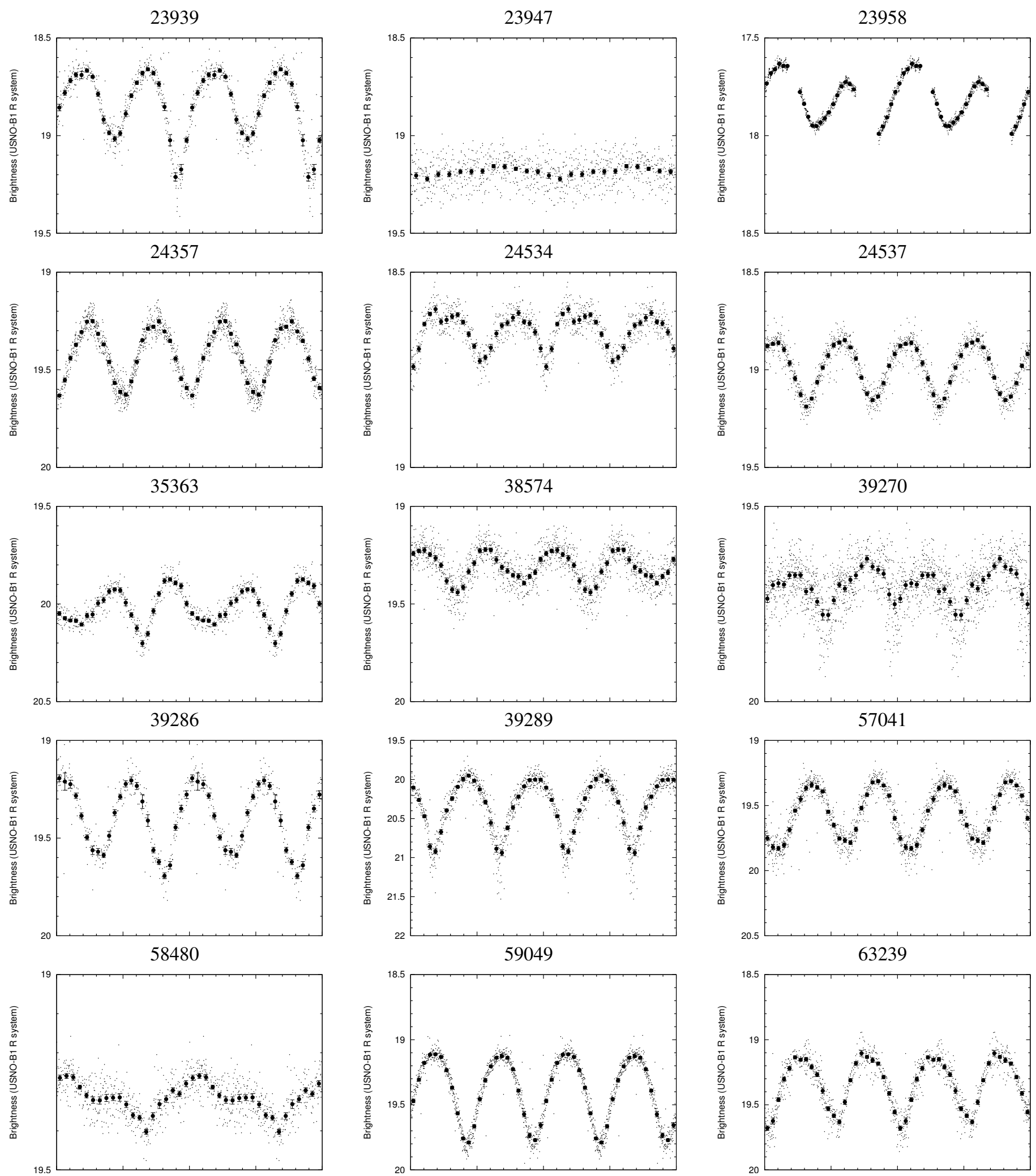

Fig. A.1. continued. 
Gy. M. Szabó et al.: K2 photometry of Jupiter Trojan asteroids
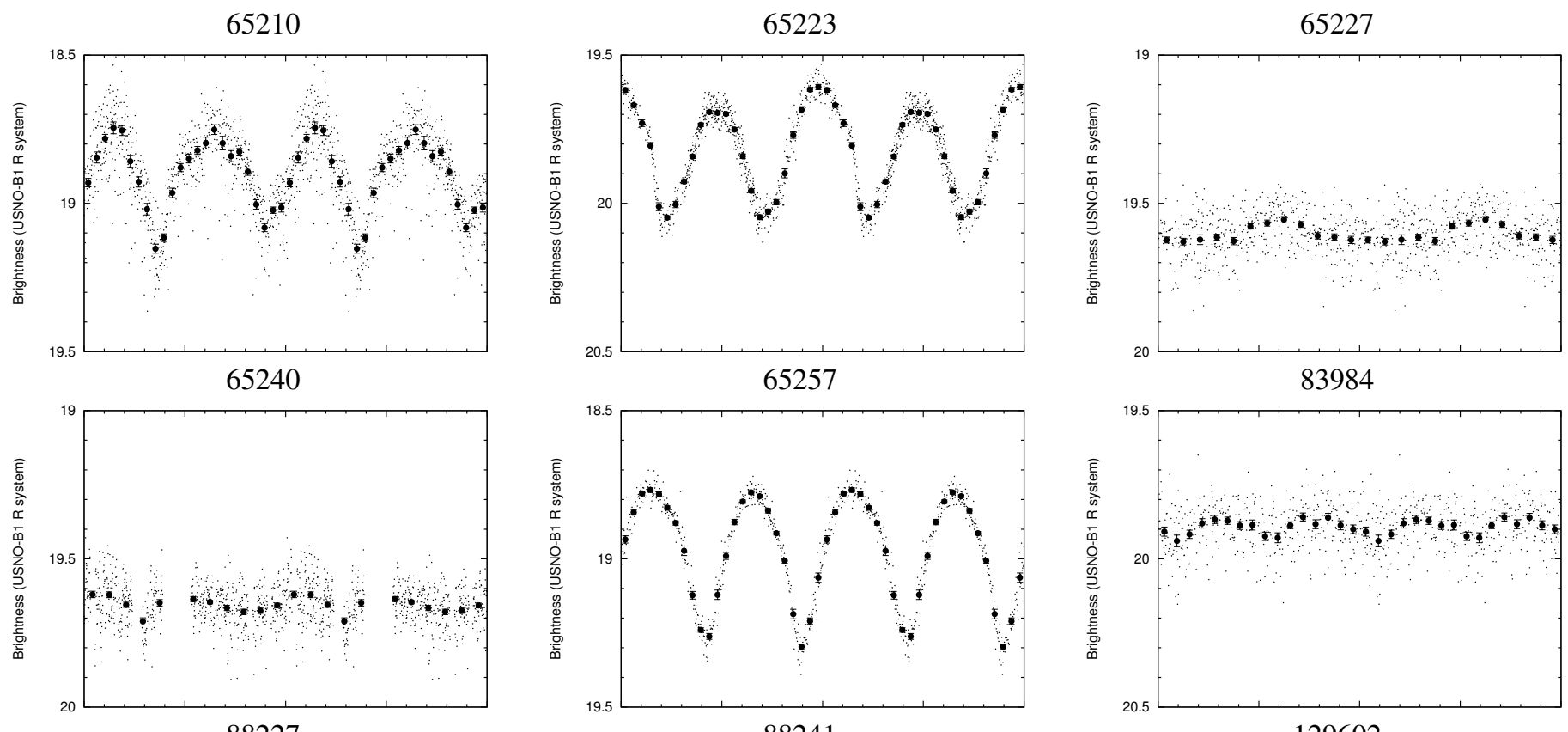

88227
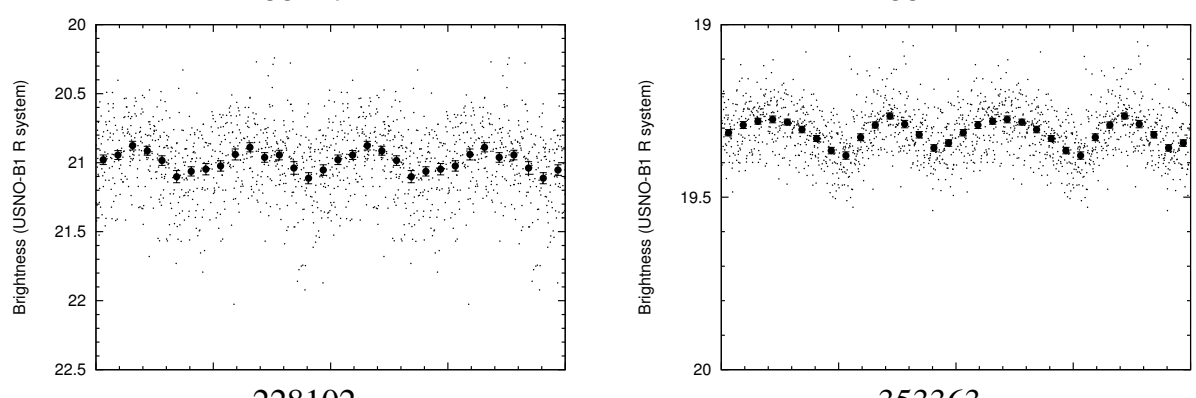

129602
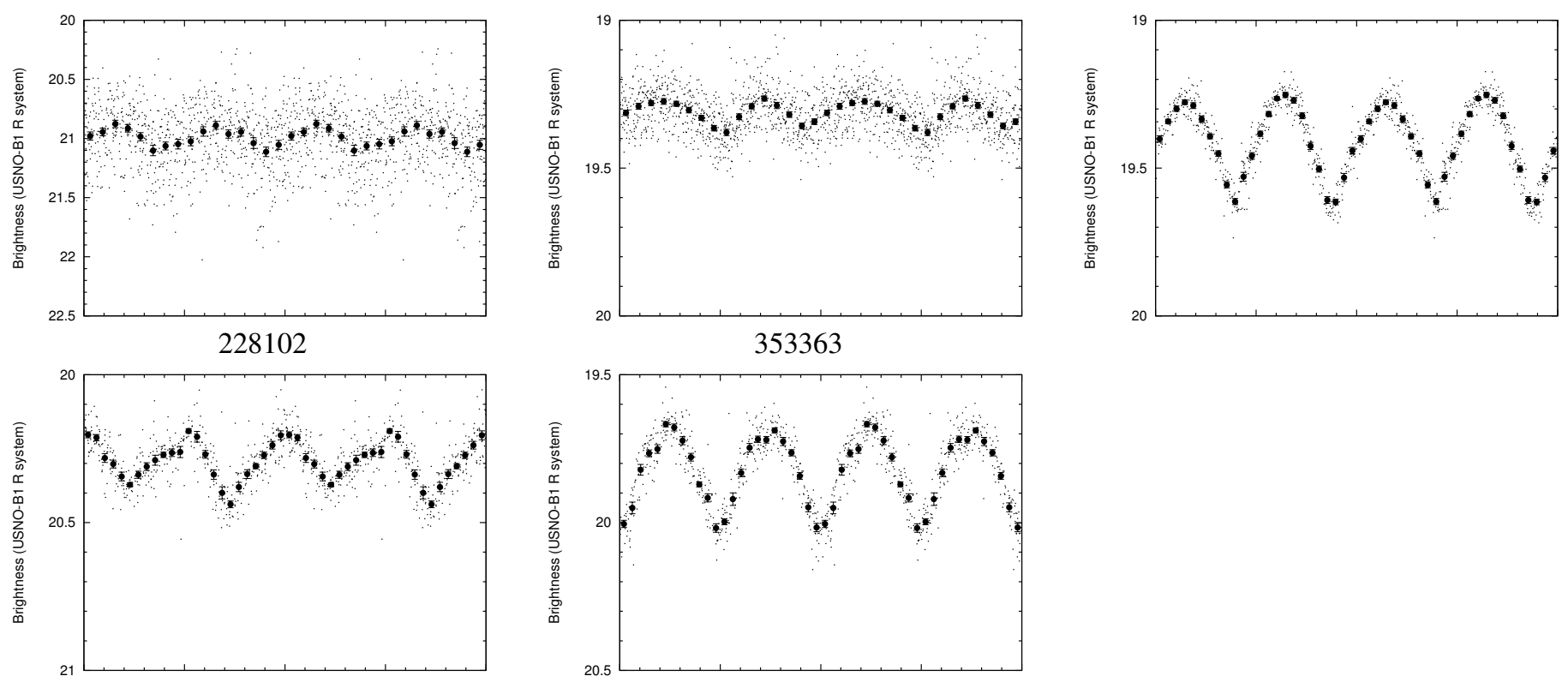

Fig. A.1. continued. 